\title{
Do Stemness Markers in Oral Erythroplakia Have a Role in Malignant Transformation: A Comprehensive Review
}

\author{
Shankargouda Patil ${ }^{1}$, Saranya Varadarajan ${ }^{2}$, Prasanna Nichat ${ }^{3}$, Johara M Cruz ${ }^{4}$, Muralimani Mani ${ }^{5}$, Sachin C Sarode ${ }^{6}$, \\ Gargi S Sarode ${ }^{7}$, Vikrant R Patil ${ }^{8}$, Zohaib Khurshid ${ }^{9}$, Muhammad S Zafar ${ }^{10}$
}

\begin{abstract}
Oral squamous cell carcinoma (OSCC) —one of the most common neoplasms of the head and neck region—accounts for $90 \%$ of cancer-related deaths. It is a well-known fact that cancer stem cells (CSCs) play a vital role in tumor progression, metastasis, drug and radiation resistance, and recurrence. Potentially malignant disorders are a group of lesions that demonstrate epithelial dysplasia and are more likely to precede squamous cell carcinoma of the oral cavity. Among the various potentially malignant disorders, erythroplakia, a non-scrapable red lesion of the oral mucosa, is the most aggressive exhibiting severe dysplasia. Carcinogenesis is a multistep and complex process commencing from exposure of carcinogen to development of malignancy. Among the various pathogenic mechanisms of carcinogenesis, the role of CSCs in the malignant transformation of erythroplakia has been less explored. With the available information, the present review aims to discuss the stemness markers associated with the erythroplakia and to establish its association with erythroplakia and the early onset of OSCCs.

Keywords: Cancer stem cells, Carcinogenesis, Epithelial Dysplasia, Erythroplakia, Malignant transformation, Oral squamous cell carcinoma.

World Journal of Dentistry (2021): 10.5005/jp-journals-10015-1873
\end{abstract}

\section{INTRODUCTION}

Oral cancer is the major cause of cancer-related deaths in Asian countries. Among the head and neck cancers (HNCs), oral squamous cell carcinoma (OSCC) accounts for nearly $90 \%$ mortality rate. ${ }^{1,2}$ Oral precancerous lesions or "potentially malignant disorders" are pathogenic epithelial lesions that are more likely to precede OSCC..$^{1-4}$ Thus, there is a necessity to study the pathogenic mechanisms associated between potentially malignant disorders and OSCCs for early diagnosis and formulation of precise therapeutic strategies. Oral epithelial dysplasia (OED) is a class of oral pathologies describing the dysplastic changes in the oral mucosa. ${ }^{5-7}$ Potentially malignant disorders such as leukoplakia and erythroplakia; malignancy such as invasive OSCC are the conditions that demonstrate characteristic dysplastic changes seen in OED. ${ }^{1,3,8-11}$ Oral epithelial dysplasia is also seen in some chronic ulcers and papillary leukoplakias. ${ }^{3,9,11}$ Oral epithelial dysplasia is not only a diagnostic tool for premalignancy but also indicates the increased risk of malignant transformation. ${ }^{1-3,12}$ However, due to the increased variability in defining the histopathologic criteria to define dysplasia, there is a need for a set of well-defined markers that could be used for the diagnosis of OEDs, their malignant transformation potential, and the molecular mechanisms associated with both OEDS and oral cancers. Especially stem cell markers expressed by dysplastic cells of potentially malignant lesions in malignant transformation, tumor progression, and metastasis have been less explored. An overview of the role of cancer stem cells (CSCs) in the malignant transformation of erythroplakia is depicted in Figure 1. The present review aimed to discuss the stemness markers associated with OEDs especially erythroplakia and to establish its association with erythroplakia and early onset of OSCCs.

\section{Cancer Stem Cells and Stemness Markers}

Stem cells are defined as a distinct subset of cells identified by two major characteristics of clonogenic potential and multi-lineage differentiation into other cell types. ${ }^{13-15}$ Almost all adult tissues
${ }^{1}$ Department of Maxillofacial Surgery and Diagnostic Sciences, Division of Oral Pathology, College of Dentistry, Jazan University, Jazan, Kingdom of Saudi Arabia

${ }^{2}$ Department of Oral Pathology and Microbiology, Sri Venkateswara Dental College and Hospital, Chennai, Tamil Nadu, India

${ }^{3}$ Department of Oral Pathology, Government Dental College, Silchar, Assam, India

${ }^{4}$ Department of Prosthdontics and Implantology, Sathyabama Dental College and Hospital, Chennai, Tamil Nadu, India

${ }^{5}$ Department of Oral and Maxillofacial Surgery, Sri Venkateswara Dental College and Hospital, Chennai, India

6,7Department of Oral Pathology and Microbiology, Dr DY Patil Dental College and Hospital, Dr DY Patil Vidyapeeth, Pune, Maharashtra, India ${ }^{8}$ Biogenre Private Limited, Pune, Maharashtra, India

${ }^{9}$ Department of Prosthodontics and Dental Implantology, College of Dentistry, King Faisal University, Al-Ahsa, Kingdom of Saudi Arabia

${ }^{10}$ Department of Restorative Dentistry, College of Dentistry, Taibah University, Al Madina Al Munawara, Kingdom of Saudi Arabia

Corresponding Author: Shankargouda Patil, Department of Maxillofacial Surgery and Diagnostic Sciences, Division of Oral Pathology, College of Dentistry, Jazan University, Jazan, Kingdom of Saudi Arabia, Phone: +966 50763 3755, e-mail:dr.ravipatil@gmail.com How to cite this article: Patil S, Varadarajan S, Nichat P, et al. Do Stemness Markers in Oral Erythroplakia Have a Role in Malignant Transformation: A Comprehensive Review. World J Dent 2021; 12(6):510-514.

Source of support: Nil

Conflict of interest: None

contain a pool of adult stem cells which on receiving external signals for growth and repair, mitosis, and differentiation to give rise to terminally differentiated cells for tissue homeostasis. 15,16

Similar to the adult tissues, stem cell populations are present in tumors and are termed CSCs. These CSCs not only provide tumor 


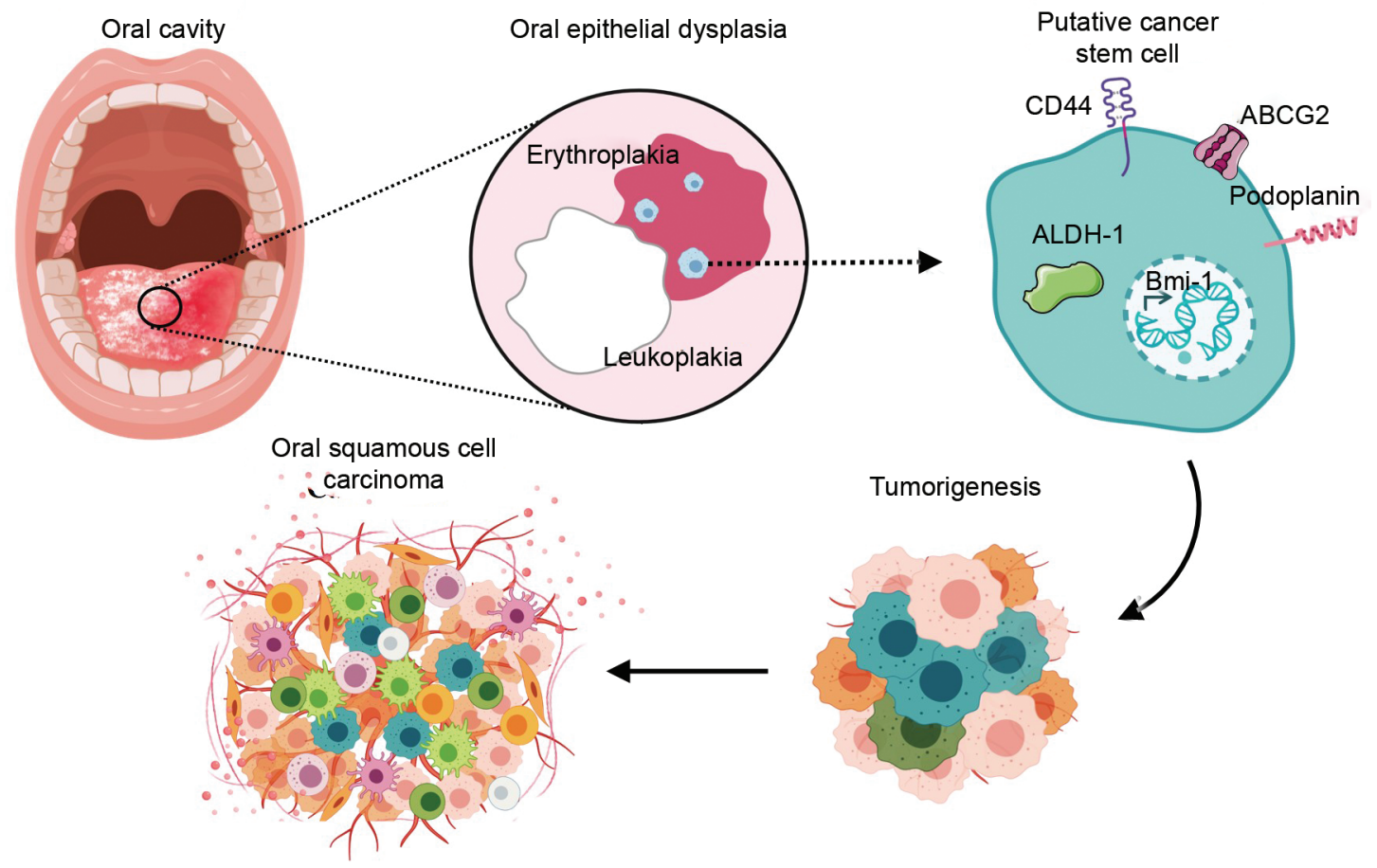

Fig. 1: An overview of the role of cancer stem cells in the malignant transformation of erythroplakia

heterogeneity but also play a vital part role in tumor progression, metastasis, drug and radiation resistance, and recurrence. ${ }^{6,15,17}$ As tissue-resident stem cells, CSCs are also responsible for continuous self-renewal and self-propagation over a long period. . $^{15,18-20}$

Most of the tumors including OSCCs have been demonstrated to have a distinct population of special tumor cells which are positive for CSC markers like CD44, CD133, ABCG2, and aldehyde dehydrogenase 1 (ALDH-1). ${ }^{12,21,22}$ These cells have been shown to demonstrate tumor formation in animal transplantation studies which is a characteristic property of CSCs. $3,9,12,15$

\section{Stemness Markers in OEDs and Oral Erythroplakia (OE)} Well-characterized CSC markers such as CD24, CD44, CD133, CD271, podoplanin, and ALDH-1 are used for the identification of cells with the properties of CSCs. ${ }^{15,23}$ Among these markers, CD44, a surface glycoprotein is being highly implicated in the regulation of tumor progression, invasion, and metastatic transformations. ${ }^{1,3,8,9,24-26}$ However, there are very few studies stating the expression of CD44 and premalignant oral lesions. In a study, the decreased CD44 expression was reported in three different degrees of epithelial dysplasia in comparison with normal mucosa. ${ }^{5,18,25,27}$ The correlation between the various grades of dysplasia and downregulation of CD44 shed light on the fact that these early cellular changes are more likely to contribute to invasion and progression of early oral carcinogenesis. ${ }^{11,21,28}$ Thus, CD44 could be very useful to determine the severity of the malignancy of the oral region.

Several studies have suggested that another transmembrane glycoprotein known as "podoplanin" could be a predictive biomarker in the early onset and progression of OSCCs. ${ }^{12,16}$ Some of the studies have also reported the elevated expressions of podoplanin protein in oral lichen planus (OLP) and leukoplakia and have concluded that this protein expression can be used to predict oral carcinogenesis in potentially malignant disorders of the oral cavity. ${ }^{26,29}$ Podoplanin a mucin-type protein has been reported to be associated with a higher risk of tumorigenesis in OLP. ${ }^{11,22,30}$ The podoplanin expression has been reported to be a vital biomarker in tumorigenesis of OEDs. ${ }^{1,2,11}$ Studies have reported that $90 \%$ of OSCCs, demonstrated an increased expression of podoplanin which was confined to the invasive front of OSCCs. ${ }^{6,12}$ Another study reported $37 \%$ podoplanin expression in oral leukoplakia and $44 \%$ in OE and malignant transformation of OE was higher than leukoplakia. ${ }^{2,5,7}$ Thus, a positive expression of podoplanin therefore can be concluded as the stemness property of CSCs in OE of their clonogenic ability and can be highly correlated with the malignant transformation of OE.

ATP-binding cassette, G2 subfamily (ABCG2) is a protein known to produce resistance against multiple anticancer drugs. A special phenotype of ABCG2 is exhibited by stem cells. ${ }^{4,17,18,31,32}$ ATP-binding cassette, $G 2$ subfamily is a widely expressed stemness protein in cancers and several studies have reported its association with tumorigenesis in $\mathrm{HNCs}{ }^{10,21}$ In a retrospective study, ABCG2 and podoplanin protein expression in tissue samples of 34 cases of OE were examined histopathologically. ATP-binding cassette, G2 subfamily and podoplanin protein expressions, therefore, are likely to be deregulated in $\mathrm{OE}$.

Bmi-1 senescence and cell cycle in stem cells regulating gene are also associated with CSCs and OSCCs of the HNC region. ${ }^{5,33} \mathrm{~A}$ study reported increased density of malignant and oral potentially malignant lesions showing $A B C G 2$ and $\mathrm{Bmi}-1$ expression and cell lines derived from them. ${ }^{8}$

A few studies have reported ALDH-1 as a marker for CSCs in adult cancers ALDH-1 as it is involved in the early differentiation of stem cells. ${ }^{18,23,24,34}$ A few studies have shown that ALDH-1 and Bmi-1 are not only characterized in CSCs of HNC tumors but also are involved in the maintenance of clonogenicity and tumorigenicity of CSCs present in OSCC tumors. ${ }^{25,28}$ In a retrospective study, the 
expressions of ALDH-1 and Bmi-1 were assessed in 34 cases of OE and subsequent oral carcinomas. ${ }^{12}$ They reported a significant correlation between ALDH-1 and Bmi-1 expression and a highly cancerous lesion of OE that developed into multiple tumors.

\section{Erythroplakia the Major Risk Factor of OSCC}

The incidence and prevalence of OSCC have increased several folds in the past few decades, especially in several countries of the Asian continent. ${ }^{1,2,31}$ Hence, current research focus is on the development of techniques that would aid early diagnosis of OSCC or potentially malignant disorders to prevent cancer spread. Clinical lesions such as oral submucous fibrosis (OSMF), leukoplakia, erythroplakia, and OLP reveal dysplastic changes. ${ }^{1,4}$ These premalignant conditions provide huge scope to assess the underlying cellular and molecular events, which would aid in early diagnosis thereby improving prognosis.

Among the various potentially malignant disorders, $\mathrm{OE}$ is a redcolored and extremely aggressive oral lesion exhibiting increased dysplastic changes. It is prevalent in males with an age predilection of 50-70 years. The reported point prevalence rate of $O E$ is 1 in 2,500 adults. ${ }^{19,35}$ The most common site of occurrence of $O E$ includes the soft palate, mouth floor, lateral part of the tongue, and retromolar pad. ${ }^{5,30}$ The lesion is usually asymptomatic, however, few patients present with burning sensation as a symptom. ${ }^{22}$ The condition has a higher malignant transformation rate of $90 \% .{ }^{35}$ Thus, erythroplakia is one of the most common predecessors of OSCC. However, there is very limited literature available on the molecular and cellular mechanisms involved in the pathogenesis of malignant transformations of OE. Considering the prognosis OE has shown to have a higher malignant transformation rate in comparison with leukoplakia. ${ }^{2,5,8-10,36}$ In a study, analysis of biopsies from 65 patients with erythroplakia revealed that $51 \%$ of cases were with invasive OSCC and $40 \%$ were having severely dysplastic epithelium whereas only $9 \%$ were having a less lethal form of dysplasia. ${ }^{8,9,36,37}$ Figure 2 represents the association of OED and the development of OSCC. Figure 3 represents the key molecular markers of CSCs in OE which could play a role in malignant transformation.

\section{Clinical Implications and Cutting the Roots of OSCCs by Targeting Erythroplakia CSCs}

Oral squamous cell carcinoma is initiated from cells that have acquired genetic alterations or mutations, such as activation and amplification of cancer-related genes and suppression of tumor suppressor genes. ${ }^{1,733}$ Though, uncharacteristic gene expression patterns are supposedly pertinent to tumorigenesis of the oral mucosa, deregulated functions of some of the key promoters, interrelated with or moderated by those genes, show critical roles in the development of malignant transformation and promotion of cancer progression. ${ }^{7,38}$ Identification of predictable, sensitive, and reproducible biomarkers that would aid in the evaluation of perils of cancer progression is the need of the hour. Contemporary advancement in sophisticated technologies, comprising proteomics of the salivary components, analysis of miRNA, a singlenucleotide deviation revealing in some precisely known genes, and genome-wide association studies, would highly promote identifying and developing a distinct set of markers that will be used to predict and treat the OSCCs in their early stages. With the



Fig. 3: Biomarkers of oral erythroplakia. Graphical representation of possible key molecular markers identifying cancer stem cells in oral erythroplakia which could imply OSCC development in oral erythroplakia patients. OE, oral erythroplakia; Podoplanin, A transmembrane receptor glycoprotein; ABCG2, ATP-binding cassette superfamily G member 2; CD44, homing cell adhesion molecule; Bmi-1, polycomb group RING finger protein 4; ALDH-1, aldehyde dehydrogenase 1

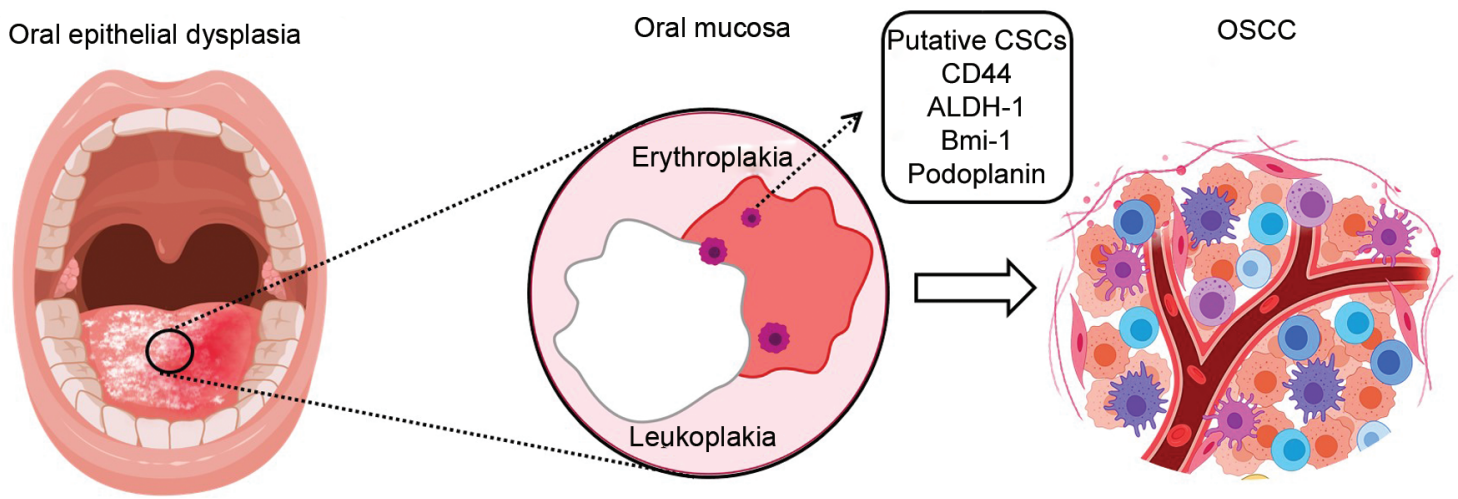

Fig. 2: Oral epithelial dysplasia and development of oral squamous cell carcinoma. Depiction of oral epithelial dysplasia showing two distinct patches; oral leukoplakia (white patch) and oral erythroplakia (red patch) and putative cancer stem cell markers in oral erythroplakia likely to be responsible for the development of squamous cell carcinoma. OSCC: Oral squamous cell carcinoma 
regular histopathologic examinations, these validated biomarkers would bring about a better diagnosis and prospects for successful therapies when the tumor progression can be successfully ceased. This would further shed light on the molecular pathways of OSCCs and would aid in the identification of novel target genes for molecular therapies.

It is a well-known fact that early diagnosis and treatment of potentially malignant disorders can prevent carcinogenesis. The prime time for medical intervention is the point where CSC-like cells start appearing. Transformation rates are highly dependent upon the presence of CSC-like cells so a more detailed analysis is needed of stemness and CSCs in oral precancerous lesions. The severity of dysplastic changes in premalignant lesions are concomitant with inferior clinical consequences, but more demanding approaches are required to envisage the tumor-forming prospects of these premalignant lesions concerning CSCs and stemness markers. Tumors frequently encompass ordered provisions of gene-wide or epigenetic divergent cancerous and non-cancerous cellular processes. ${ }^{4,14,15,39}$ In these instances, some speculative phenotypes of tumor-forming cells are being identified through exhaustive experimental work with single-cell suspensions from tumors, xenotransplantation studies, and phenotypic cell-surface antigens. The attainment of a genetic makeover to the tumor-forming ability by CSCs is a collective route that would be apparent in premalignant tissues. The relationships between proclaimed stem cell markers with these oral lesions and oral epithelial tumorigenicity have to be studied thoroughly.

Since CSCs have been implicated in several cancers much importance is being given to CSCs and the molecular pathogenesis correlating the behavior of these cells and the aggression of cancers of the HNC region including OSCCs. Most of the recent in vivo studies have revealed that the development of a tumor is sustained by a robust population of CSCs. Although the differentiating tumor cells contribute to tumor development; it is still indeterminate whether all types of tumors hold such a tiered association of tumor-growth, and development and if all existing cancer cells exhibit long-lasting self-renewing capability as CSCs. ${ }^{14,15}$

The association between CSCs and tumor niche is still poorly understood. New approaches are necessary to evaluate the CSCs and associated markers at various points of cancer development and metastasis, also to delineate their role in cancer progression. Additionally, there is a need for the development of experimental settings that simulate the entire microenvironment with CSCs to assess tumor development and establish the molecular mechanisms involved in the correlation between premalignant lesions, CSCs, and malignant transformations.

\section{Conclusion}

A considerable understanding of the mechanisms regulating CSCs in aggressive oral precancerous lesions such as erythroplakia and their radiation- and drug resistance is needed to prevent malignant transformation. Novel treatment approaches must focus on novel diagnostic and prognostic modalities to combine conventional therapeutic molecules that specifically target CSCs in the premalignant lesions or their genetic regulators.

\section{References}

1. Scully C, Sudbø J, Speight PM. Progress in determining the malignant potential of oral lesions. J Oral Pathol Med 2003;32(5):251-256. DOI: 10.1034/j.1600-0714.2003.00108.x.
2. Olinici $D$, Cotrutz $C E$, Mihali CV, et al. The ultrastructural features of the premalignant oral lesions. Rom J Morphol Embryol 2018;59(1): 243-248. http://www.ncbi.nlm.nih.gov/pubmed/29940634.

3. Reichart PA, Philipsen HP. Oral erythroplakia—a review. Oral Oncol 2005;41(6):551-561. DOI: 10.1016/j.oraloncology.2004.12.003.

4. Sudbo J, Reith A. Which putatively pre-malignant oral lesions become oral cancers?. Clinical relevance of early targeting of high-risk individuals. J Oral Pathol Med 2003;32(2):63-70. DOI: 10.1034/j.16000714.2003.00054.x.

5. Bryne M, Reibel J, Mandel U, et al. Expression of mucin type carbohydrates may supplement histologic diagnosis in oral premalignant lesions. J Oral Pathol Med 1991;20(3):120-125. DOI: 10.1111/j.1600-0714.1991.tb00904.x.

6. Holmstrup P, Thorn JJ, Rindum J, et al. Malignant development of lichen planus-affected oral mucosa. J Oral Pathol Med 1988;17(5): 219-225. DOI: 10.1111/j.1600-0714.1988.tb01528.x.

7. Sacks PG. Cell, tissue and organ culture as in vitro models to study the biology of squamous cell carcinomas of the head and neck. Cancer Metastasis Rev 1996;15(1):27-51. DOI: 10.1007/BF00049486.

8. Dalley AJ, Pitty LP, Major AG, et al. Expression of ABCG 2 and Bmi-1 in oral potentially malignant lesions and oral squamous cell carcinoma. Cancer Med 2014;3(2):273-283. DOI: 10.1002/cam4.182.

9. Woo S-B. Oral epithelial dysplasia and premalignancy. Head Neck Pathol 2019;13(3):423-439. DOI: 10.1007/s12105-019-01020-6.

10. Bhatia A, Burtness B. Novel molecular targets for chemoprevention in malignancies of the head and neck. Cancers (Basel) 2017;9(12):113. DOI: 10.3390/cancers9090113.

11. Feng J-Q, Xu Z-Y, Shi L-J, et al. Expression of cancer stem cell markers ALDH1 and Bmi1 in oral erythroplakia and the risk of oral cancer. J Oral Pathol Med 2013;42(2):148-153. DOI: 10.1111/j.1600-0714.2012. 01191.x.

12. Feng J-Q, Mi J-G, Wu L, et al. Expression of podoplanin and ABCG2 in oral erythroplakia correlate with oral cancer development. Oral Oncol 2012;48(9):848-852. DOI: 10.1016/j.oraloncology.2012. 03.015 .

13. McNamara KK, Kalmar JR. Erythematous and vascular oral mucosal lesions: a clinicopathologic review of red entities. Head Neck Pathol 2019;13(1):4-15. DOI: 10.1007/s12105-019-01002-8.

14. Desai A, Yan Y, Gerson SL. Concise reviews: cancer stem cell targeted therapies: toward clinical success. Stem Cells Transl Med 2019;8(1): 75-81. DOI: 10.1002/sctm.18-0123.

15. Nassar D, Blanpain C. Cancer stem cells: basic concepts and therapeutic implications. Annu Rev Pathol Mech Dis 2016;11(1):47-76. DOI: 10.1146/annurev-pathol-012615-044438.

16. Yang $X$, Shi $L$, Zhou Z, et al. Podoplanin and ABCG2 expression in oral erythroplakia revisited: potential evidence for cancer stem cells driving the process of field cancerization. Oral Oncol 2020;101:104368. DOI: 10.1016/j.oraloncology.2019.07.011.

17. Lumerman $H$, Freedman $P$, Kerpel $S$. Oral epithelial dysplasia and the development of invasive squamous cell carcinoma. Oral Surgery, Oral Med Oral Pathol Oral Radiol Endodontology 1995;79(3):321-329. DOI: 10.1016/S1079-2104(05)80226-4.

18. Holmstrup P. Oral erythroplakia-what is it? Oral Dis 2018;24(1-2): 138-143. DOI: 10.1111/odi.12709.

19. Sudbø J, Kildal W, Johannessen AC, et al. Gross genomic aberrations in precancers: clinical implications of a long-term follow-up study in oral erythroplakias. J Clin Oncol 2002;20(2):456-462. DOI: 10.1200/ JCO.2002.20.2.456.

20. Capocasale G, Panzarella V, Tozzo P, et al. Oral verruciform xanthoma and erythroplakia associated with chronic graft-versus-host disease: a rare case report and review of the literature. BMC Res Notes 2017;10(1):631. DOI: 10.1186/s13104-017-2952-7.

21. van der WI, Scully C. 4. Potentially malignant disorders of the oral and oropharyngeal mucosa. Dent Update 2011;38(2):138-140. DOI: 10.12968/denu.2011.38.2.138.

22. Bánóczy J. Oral cancer and precancerous lesions. Fogorv Sz 1997;90(Spec No:27):PMID: 9170723. http://www.ncbi.nlm.nih.gov/ pubmed/9170723. 
23. Mithani S, Mydlarz W, Grumbine F, et al. Molecular genetics of premalignant oral lesions. Oral Dis 2007;13(2):126-133. DOI: 10.1111/j.1601-0825.2006.01349.x.

24. Shi L, Jiang W, Liu W. Retrospective analysis of oral erythroplakia focused on multiple and multifocal malignant behavior. Oral Dis 2019;25(7):1829-1830. DOI: 10.1111/odi.13144.

25. Yang X, Liu W. Bibliometric analysis of the top-cited articles on oral erythroplakia and leukoplakia. J Oral Pathol Med 2019;48(6):505-506. DOI: 10.1111/jop.12863.

26. Nikitakis NG, Rassidakis GZ, Tasoulas J, et al. Alterations in the expression of DNA damage response-related molecules in potentially preneoplastic oral epithelial lesions. Oral Surg Oral Med Oral Pathol Oral Radiol 2018;125(6):637-649. DOI: 10.1016/j.0ooo.2018.03. 006.

27. Venkat Naga SS, Shekar P, Kattappagari K, et al. Expression of cluster differentiation-44 stem cell marker in grades of oral epithelial dysplasia: a preliminary study. J Oral Maxillofac Pathol 2019;23(2):203. DOI: 10.4103/jomfp.JOMFP_308_18.

28. Liu S-Y, Feng I-J, Wu Y-W, et al. Implication for second primary cancer from visible oral and oropharyngeal premalignant lesions in betel-nut chewing related oral cancer. Head Neck 2017;39(7):1428-1435. DOI: 10.1002/hed.24777.

29. Greer RO. Pathology of malignant and premalignant oral epithelial lesions. Otolaryngol Clin North Am 2006;39(2):249-275. DOI: 10.1016/j. otc.2005.11.002.

30. Villa A, Villa C, Abati S. Oral cancer and oral erythroplakia: an update and implication for clinicians. Aust Dent J 2011;56(3):253-256. DOI: 10.1111/j.1834-7819.2011.01337.x.
31. Feng J, Zhou Z, Shi L, et al. Cancer stem cell markers ALDH1 and Bmi1 expression in oral erythroplakia revisited: Implication for driving the process of field cancerization. J Oral Pathol Med 2020;49(1):96-99. DOI: 10.1111/jop.12955.

32. Rhodus NL, Kerr AR, Patel K. Oral cancer. Dent Clin North Am 2014;58(2):315-340. DOI: 10.1016/j.cden.2013.12.004.

33. Boy SC. Leukoplakia and erythroplakia of the oral mucosa--a brief overview. SADJ 2012;67(10):558-560. http://www.ncbi.nlm.nih.gov/ pubmed/23957095.

34. Nakahata K, Uehara S, Nishikawa S, et al. Aldehyde dehydrogenase 1 (ALDH1) Is a potential marker for cancer stem cells in embryonal rhabdomyosarcoma. PLoS One 2015;10(4):e0125454. DOI: 10.1371/ journal.pone.0125454.

35. Mortazavi H, Baharvand M, Mehdipour M. Oral potentially malignant disorders: an overview of more than 20 entities. J Dent Res Dent Clin Dent Prospects 2014;8(1):6-14. DOI: 10.5681/joddd.2014. 002.

36. Smith J, Rattay T, McConkey C, et al. Biomarkers in dysplasia of the oral cavity: a systematic review. Oral Oncol 2009;45(8):647-653. DOI: 10.1016/j.oraloncology.2009.02.006.

37. Shafer WG, Waldron CA. Erythroplakia of the oral cavity. Cancer 1975;36(3):1021-1028.DOI: 10.1002/1097-0142(197509)36:3<1021::AIDCNCR2820360327>3.0.CO;2-W.

38. Jin G. Using biomarkers to detect oral cancer holds potential for saving lives when the cancer is most curable. Biomark Med 2010;4(6):835-838. DOI: $10.2217 / \mathrm{bmm} .10 .105$.

39. Batlle E, Clevers H. Cancer stem cells revisited. Nat Med 2017;23(10):1124-1134. DOI: 10.1038/nm.4409. 